\title{
DETERMINATION OF CONTAMINATION DISTANCE FOR BOREHOLES IN OKIGWE ZONE, IMO STATE NIGERIA
}

\author{
Uchendu E.E, \\ Department Of Agric. Engineering \\ Federal Polytechnic Nekede \\ Imo State, Nigeria
}

\begin{abstract}
Groundwater contaminants in Okigwe zone in Imo state made up of six local government areas were investigated. Four (4) groundwater samples were collected from each local government area. This amounted to twentyfour groundwater samples. These samples were collected randomly from sites close to septic tanks and to avoid contamination from tanks, the samples were collected at the well head, before water enters into storage tanks. The samples were stored in a sterilized $250 \mathrm{ml}$ bottles and then taken to the laboratory for analysis. The chemical parameters were determined using a $\mathrm{HA}-\mathrm{CH}$ 44600-00 and using standard methods as contained in Chessbourgh (2014). These samples were refrigerated and analyzed within $24 \mathrm{~h}$. All plastics and glass wares utilized were pre-washed with detergent water solution, rinsed with tap water and soaked for $48 \mathrm{~h}$ in $50 \% \mathrm{HNO3}$ then rinsed thoroughly with distilled- deionized water. These results were used to ascertain the levels of groundwater contamination in Okigwe zone. An equation was generated from the chemical parameters using SPSS and E-view softwares to estimate the minimum allowable distance for locating borehole from sources of contamination in Okigwe zone in Imo state Nigeria. The minimum allowable distance calculated for groundwater from sources of contamination (septic tanks) is 15.81meters.
\end{abstract}

Keywords: $\quad$ Groundwater, Pollution, Contaminants, Septic tank

\section{INTRODUCTION}

Groundwater is important for livelihood even for the socioeconomic development of an environment. Nickson et al. (2005) established that approximately one third of the world's population use groundwater for drinking, domestic and industrial purposes. More than $70 \%$ of the wastes generated in Imo state are

\author{
Madubuike C.N, Okereke C.D, Okereke N.A.A \\ Department Of Agric. Engineering \\ Federal University Of Technology Owerri \\ Imo State, Nigeria
}

discharged into the environment without any form of treatment. These wastes are dissolved by precipitation and irrigation water and produce contaminants which infiltrate into the soil and end up into the aquifers. Others flow through the surface water into the groundwater and start circulating from there. Once contaminated, it is difficult, if not impossible, for the water quality to be restored not even by stopping the pollution from source (Remarkrishnaiah et al., 2009). Ijeh and Onu (2013) showed that the deterioration of groundwater quality in Imo state can be attributed to pollution from anthropogenic and natural sources. Nwachukwu (2014) showed that most dumpsites are usually haphazardly located without careful consideration of environmental and public health.

Hundreds of people die every year from water borne diseases. The rate of deaths recorded over the last ten years in Imo state has given rise to scrutinizing the level of water quality in the state. Human activities have contributed in no small measure to the pollution of groundwater and have become less suitable for drinking, domestic and agricultural purposes. Indiscriminate dumping of refuse has deteriorated the ecosystem and the environment.

The sources of these wastes include food processing preservations, dumpsites, septic tanks, acid water recharge, abattoirs, urban runoff, oil spillage, pesticides, insecticides, herbicides, fertilizers, condemn batteries, dry cleaning chemicals, cemeteries, urine and hospital wastes. The presents of these contaminants in groundwater has caused so many water borne diseases like cholera, typhoid and hepatitis. Kidney disease, cancer of skin, bladder and lung diseases, neurological disorder, muscular weakness, pigmentation changes, skin thickening, loss of appetite and nausea may result from consuming contaminated water (Feachen et al., 1998). The trend may jeopardize the global zest 
towards millennium development goal of health for all, if unchecked. There is need for proactive action both at national and international levels to restore the quality of groundwater and be able to sustain and transfer to the next generation. Waste management to avoid contaminating both surface and subsurface water should be a global concern and regular check of water quality should be paramount in this $21^{\text {st }}$ century. . Treatment of pollution these days are multifaceted in that new emerging pollutants are being discovered every year. Old treatment methods may no longer be effective to combat these pollutants. Conventional methods for removing groundwater contaminants are either becoming inadequate to meet current stringent regulatory effluents limit or are increasing in cost (Ragasulocham and Prealthy, 2016).

\section{MATERIALS AND METHODS}

Twenty-four groundwater samples were collected from boreholes located around the Okoigwe zone Imo state, South Eastern Nigeria. The samples were stored in a sterilized $250 \mathrm{ml}$ bottles and then taken to the laboratory for analysis. The chemical parameters were determined using a HA-CH 44600-00 using standard methods as contained in Chessbourgh (2014). These samples were refrigerated and analyzed within $24 \mathrm{~h}$. All plastics and glass wares utilized were pre-washed with detergent water solution, rinsed with tap water and soaked for $48 \mathrm{~h}$ in $50 \% \mathrm{HNO} 3$ then rinsed thoroughly with distilleddeionized water. These results were used to ascertain the levels of groundwater contamination in Okigwe zone. An equation was generated from the chemical parameters using SPSS and E-view softwares to estimate the miniimum allowable distance for locating borehole from sources of contamination in Okigwe zone in Imo state Nigeria. The minimum allowable distance calculated for groundwater from sources of contamination is 15.81 meters. The result showing possible sources of contaminants was shown in Table 1. They were then air-dried in a dust free environment. The results were presented in Table 2.

\section{RESULTS AND DISCUSSION}

The results of groundwater chemical parameters for Okigwe zone in Imo state were shown in table 2. The Table below shows the values of borehole water samples and their distances from possible sources of contamination. The distances are in meters measured with the help of a meter rule. The lowest value is $12 \mathrm{~m}$ and the highest value is $34 \mathrm{~m}$.

Table 1: Borehole Water Samples and Their Distances from Possible Sources of Contamination.

\begin{tabular}{|c|c|c|c|}
\hline LGA & Area & $\begin{array}{l}\text { Distance from closet } \\
\text { potential source of } \\
\text { contamination }(\mathrm{m})\end{array}$ & $\begin{array}{l}\text { Closest source of } \\
\text { contamination. }\end{array}$ \\
\hline \multirow[t]{4}{*}{ Ehime Mbano } & Umuezeala & 28 & Septic tank \\
\hline & Umueze II & 19 & Septic tank \\
\hline & Agbja & 14 & Septic tank \\
\hline & Umunakuru & 28 & Septic tank \\
\hline \multirow[t]{4}{*}{ Isiala Mbano } & Anara & 12 & Septic tank \\
\hline & Amaraku & 17 & Septic tank \\
\hline & Ugiri & 25 & Septic tank \\
\hline & Umunkwo & 20 & Septic tank \\
\hline \multirow[t]{4}{*}{ Onuimo } & Umuduru & 32 & Septic tank \\
\hline & Okwe & 28 & Septic tank \\
\hline & Okwelle & 15 & Septic tank \\
\hline & Umuopara & 17 & Septic tank \\
\hline \multirow{4}{*}{ Obowo } & Umunachi & 20 & Septic tank \\
\hline & Achingala & 34 & Septic tank \\
\hline & Avutu & 20 & Septic tank \\
\hline & Alaike & 22 & Septic tank \\
\hline \multirow[t]{4}{*}{ Ihitte Uboma } & Isinwaeke & 12 & Septic tank \\
\hline & Amakohia & 31 & Septic tank \\
\hline & Umuihi & 20 & Septic tank \\
\hline & Aboeke & 33 & Septic tank \\
\hline
\end{tabular}




\section{International Journal of Engineering Applied Sciences and Technology, 2021}

Vol. 6, Issue 2, ISSN No. 2455-2143, Pages 17-25

Published Online June 2021 in IJEAST (http://www.ijeast.com)

\begin{tabular}{|l|l|l|l|}
\hline Okigwe & Ope & 24 & Septic tank \\
\hline & Ubahaa & 30 & Septic tank \\
\hline & Umuka & 20 & Septic tank \\
\hline & Umuokpara & 18 & Septic tank \\
\hline
\end{tabular}

The Table below shows the values of chemical values from selected boreholes in Okigwe zone. The chemical parameters are $\mathrm{Ca}, \mathrm{Zn}, \mathrm{Pb}, \mathrm{Fe}, \mathrm{Mg}, \mathrm{Na}, \mathrm{SO}_{4}, \mathrm{PO}_{4}, \mathrm{Ci}$, and $\mathrm{NO}_{3}$. The second row of the Table 2 shows the world Health Organization (WHO) and Nigeria Standard for Drinking Quality Water (NSDQW) permissible values for comparison of results.

Table 2: Chemical Parameters Values of Sample before Treatment

\begin{tabular}{|c|c|c|c|c|c|c|c|c|c|c|c|}
\hline LGA & Sample Loc & $\mathrm{Ca}$ & $\mathrm{Zn}$ & $\mathrm{Pb}$ & $\mathrm{Fe}$ & $\mathrm{Mg}$ & $\mathrm{Na}$ & $\mathrm{SO}_{4}$ & $\mathrm{PO}_{4}$ & $\mathrm{CI}$ & $\mathrm{NO}_{3}$ \\
\hline & WHO & 200 & 5.0 & $10 . \mu \mathrm{g}$ & 0.3 & 50 & 200 & 250 & 10 & 250 & 50 \\
\hline & NSDQW & 200 & 5.0 & $10 . \mu \mathrm{g}$ & 0.3 & 50 & 200 & 100 & 5 & 250 & 10 \\
\hline \multirow{4}{*}{ Ehime } & Umuezeala & 41.2 & 0.8 & Nil & 0.43 & 3.43 & 2.48 & 3.5 & 5.0 & 12.4 & 7.0 \\
\hline & Umueze II & 57.8 & 0.7 & Nil & 0.29 & 5.04 & Nil & 5.1 & 2.8 & 11.7 & 3.5 \\
\hline & Agbja & 53.4 & 0.6 & Nil & 0.8 & 0.04 & 1.55 & 2.6 & 2.3 & 9.3 & 4.6 \\
\hline & Umunakuru & 39.6 & 1.1 & 0.02 & 0.22 & 2.02 & 1.59 & Nil & 3.4 & 7.6 & 5.9 \\
\hline \multirow[t]{4}{*}{ Isiala } & Anara & 33 & 1.2 & 0.02 & 0.42 & 3.2 & 2.34 & Nil & 3.6 & 12.5 & 11.8 \\
\hline & Amaraku & 35.2 & 1.1 & Nil & 0.38 & 2.9 & 2.32 & 11.9 & 5.1 & 5.4 & 3.8 \\
\hline & Ugiri & 37.8 & 0.8 & 0.15 & 0.2 & 1.28 & Nil & 12.7 & 4.8 & 6.3 & 2.7 \\
\hline & Umunkwo & 40.8 & 0.7 & Nil & 0.4 & 1.39 & 2.04 & 2.1 & 1.7 & 6.8 & 4.0 \\
\hline \multirow[t]{4}{*}{ Ihitte } & Isinwaeke & 41.8 & 0.8 & Nil & 0.9 & 2.44 & 4.05 & Nil & 3.3 & 6.7 & 4.8 \\
\hline & Amakohia & 53.4 & 1.2 & 0.015 & 0.8 & 1.82 & 2.84 & 12.6 & Nil & 8.2 & 3.7 \\
\hline & Umuihi & 37 & 1.4 & Nil & 0.45 & 3.04 & Nil & 13.2 & 4.4 & 7.9 & 4.4 \\
\hline & Aboeke & 38.6 & 1.0 & Nil & 0.32 & 4.31 & 1.96 & 14.1 & 3.4 & 8.8 & 5.7 \\
\hline \multirow[t]{4}{*}{ Okigwe } & Ope & 29.2 & 0.8 & Nil & 0.4 & 2.43 & 1.34 & 6.7 & Nil & 10.3 & 6.8 \\
\hline & Ubahaa & 57.8 & 1.7 & Nil & 0.3 & 6.42 & 1.83 & 12.4 & 2.6 & 14.5 & 11.0 \\
\hline & Umuka & 65.4 & 1.8 & Nil & 0.38 & 4.31 & Nil & 4.2 & 3.1 & 8.2 & 8.7 \\
\hline & Umuokpara & 39.2 & 2.0 & Nil & 0.46 & 2.4 & 2.37 & Nil & 5.0 & 9.0 & 6.3 \\
\hline \multirow[t]{4}{*}{ Obowo } & Umunachi & 37.2 & 0.7 & Nil & 0.77 & 4.09 & Nil & 3.6 & 4.4 & 7.2 & 4.8 \\
\hline & Achingala & 38.4 & 1.1 & Nil & 0.16 & 3.05 & 2.02 & 6.3 & Nil & 4.6 & 3.7 \\
\hline & Avutu & 42.6 & 1.0 & Nil & 0.36 & 1.84 & 3.12 & 2.4 & 3.2 & 17.4 & 3.7 \\
\hline & Alaike & 44.8 & 0.7 & Nil & 0.38 & 3.24 & 3.41 & 13.2 & Nil & 8.2 & 7.2 \\
\hline \multirow[t]{4}{*}{ Onuimo } & Umuduru & 40.2 & 0.7 & Nil & 0.4 & 1.85 & 3.23 & 2.9 & Nil & 6.4 & 5.2 \\
\hline & Okwe & 42.4 & 0.5 & Nil & 0.32 & 1.04 & 4.01 & 11.8 & 2.6 & 5.6 & 3.2 \\
\hline & Okwelle & 32.2 & 0.6 & Nil & 0.3 & 2.05 & 2.01 & 2.7 & 3.8 & 6.2 & 7.2 \\
\hline & Umuopara & 34.6 & 1.0 & Nil & 0.29 & 1.06 & Nil & 3.6 & 4.7 & 4.8 & 6.3 \\
\hline
\end{tabular}

All units are in $\mathrm{mg} / \mathrm{I}$ except where stated.

Table 3 shows the treatment of these water samples with permeable reactive barrier filtration materials. The second row is for the comparison with WHO and NSDQW.

$\underline{\text { Table 4. Descriptive Statistics and Normality Test value for chemical parameters }}$ 


\section{International Journal of Engineering Applied Sciences and Technology, 2021 \\ Vol. 6, Issue 2, ISSN No. 2455-2143, Pages 17-25 \\ Published Online June 2021 in IJEAST (http://www.ijeast.com)}

\begin{tabular}{|l|c|c|c|c|c|c|c|c|c|c|c|}
\hline & $\mathrm{CA}$ & $\mathrm{CL}$ & $\mathrm{CONTD}$ & $\mathrm{FE}$ & $\mathrm{MG}$ & $\mathrm{NA01}$ & $\mathrm{NO}$ & $\mathrm{PB}$ & $\mathrm{PO} 4$ & $\mathrm{SO}$ & $\mathrm{ZN}$ \\
\hline Mean & 149.644 & 27.52000 & 83.88889 & 1.323333 & 9.799630 & 9.159259 & 21.88519 & 0.030185 & 11.91111 & 25.74111 & 4.107407 \\
\hline Median & 146.8000 & 27.86000 & 85.00000 & 1.190000 & 9.740000 & 8.710000 & 21.00000 & 0.020000 & 11.60000 & 24.50000 & 3.900000 \\
\hline Maximum & 222.6000 & 45.30000 & 124.0000 & 2.900000 & 15.56000 & 14.62000 & 43.50000 & 0.150000 & 25.40000 & 51.90000 & 6.300000 \\
\hline Minimum & 99.40000 & 9.850000 & 50.00000 & 0.700000 & 5.600000 & 5.540000 & 12.00000 & 0.000000 & 6.700000 & 8.300000 & 2.800000 \\
\hline Std. Dev. & 31.62060 & 9.673008 & 16.46753 & 0.523391 & 2.371690 & 2.443053 & 6.398059 & 0.032713 & 3.440520 & 10.15592 & 0.957933 \\
\hline Skewness & 0.336825 & -0.050166 & 0.213393 & 1.415539 & 0.277345 & 0.641003 & 1.402798 & 1.969509 & 2.160217 & 0.623054 & 0.700645 \\
\hline Kurtosis & 2.460619 & 2.172407 & 3.148471 & 4.723522 & 2.942238 & 2.916760 & 6.193488 & 7.752920 & 9.973975 & 3.136588 & 2.896573 \\
\hline & & & & & & & & & & & \\
\hline $\begin{array}{l}\text { Jarque- } \\
\text { Bera }\end{array}$ & 0.837829 & 0.781848 & 0.229713 & 12.35872 & 0.349894 & 1.856778 & 20.32845 & 42.86937 & 75.71528 & 1.767871 & 2.221098 \\
\hline Probability & 0.657760 & 0.676432 & 0.891494 & 0.002072 & 0.839501 & 0.395190 & 0.000039 & 0.000000 & 0.000000 & 0.413154 & 0.329378 \\
\hline & & & & & & & & & & & \\
\hline Sum & 4040.400 & 743.0400 & 2265.000 & 35.73000 & 264.5900 & 247.3000 & 590.9000 & 0.815000 & 321.6000 & 695.0100 & 110.9000 \\
\hline $\begin{array}{l}\text { Sum Sq. } \\
\text { Dev. }\end{array}$ & 25996.43 & 2432.744 & 7050.667 & 7.122400 & 146.2477 & 155.1812 & 1064.314 & 0.027824 & 307.7667 & 2681.708 & 23.85852 \\
\hline & & & & & & & & & & & \\
\hline $\begin{array}{l}\text { Observatio } \\
\text { ns }\end{array}$ & 27 & 27 & 27 & 27 & 27 & 27 & 27 & 27 & 27 & 27 & 27 \\
\hline
\end{tabular}

From Table 4, it could be seen that the data is not normally distributed as the mean values of the variables are so dispersed from each other in value. Their probability values show an unstable conclusion about their normality as well as the fact that some are less than 0.05 and others are greater than 0.05 . To validate the normality assumption, we take an examination of the histogram normality test below.
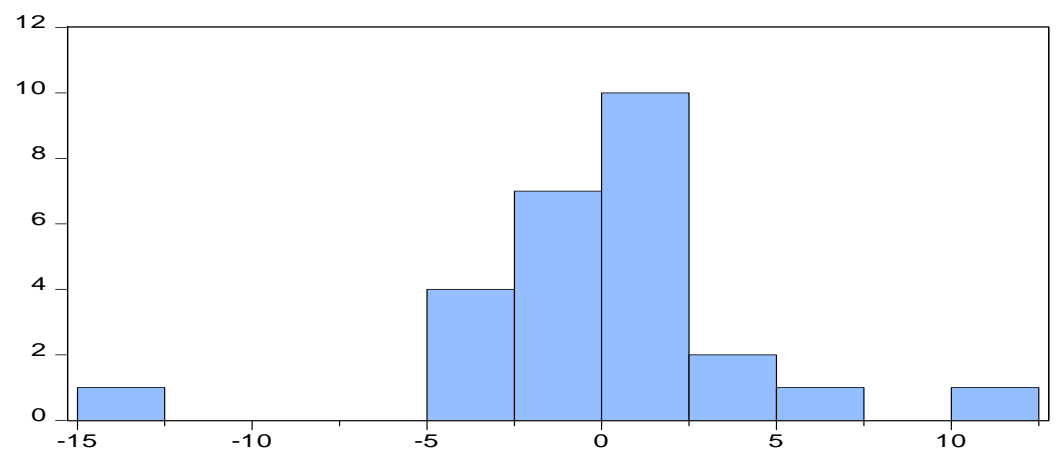

\begin{tabular}{lr}
\multicolumn{3}{l}{ Series: Residuals } \\
Sample 2 27 \\
Observations 26 \\
Mean & $1.87 e-14$ \\
Median & 0.239932 \\
Maximum & 10.16323 \\
Minimum & -12.92963 \\
Std. Dev. & 4.097552 \\
Skewness & -0.566777 \\
Kurtosis & 6.092941 \\
& \\
Jarque-Bera & 11.75550 \\
Probability & 0.002801 \\
\hline
\end{tabular}

Figure 1 Histogram Normality Test

( Source: E-views 10 Regression output.)

From the data above, the probability value (0.002801) was less than 0.05 ; hence the sample data does not satisfy normality distribution assumption as corroborated above.

Table 5 Stationarity (Unit Root) Test.

\begin{tabular}{|l|l|l|l|l|l|}
\hline Variables & $\begin{array}{l}\text { Adf } \\
\text { Stat. (level) }\end{array}$ & $\begin{array}{l}5 \% \\
\text { Critical value }\end{array}$ & $\begin{array}{l}\text { Adf } \\
\text { Stat.(1 }{ }^{\text {st } d i f f)}\end{array}$ & $\begin{array}{l}5 \% \\
\text { Critical value }\end{array}$ & Remark \\
\hline CA & $-4.935302^{*}$ & -3.711457 & -9.669154 & -2.986225 & $\mathrm{I}(0)$ \\
\hline CL & $-4.279067^{*}$ & -2.981038 & -6.721036 & -2.986225 & $\mathrm{I}(0)$ \\
\hline CONTD & -2.514514 & -2.981038 & $-6.343421^{*}$ & -2.986225 & $\mathrm{I}(1)$ \\
\hline FE & $-3.524563^{*}$ & -2.981038 & -5.640724 & -2.986225 & $\mathrm{I}(0)$ \\
\hline MG & $-4.185471^{*}$ & -2.981038 & -7.543754 & -2.986225 & $\mathrm{I}(0)$ \\
\hline NA01 & $-3.384307^{*}$ & -2.981038 & -6.718346 & -2.986225 & $\mathrm{I}(0)$ \\
\hline NO3 & $-4.088000^{*}$ & -2.981038 & -8.629529 & -2.986225 & $\mathrm{I}(0)$ \\
\hline PB & $-3.533289^{*}$ & -2.981038 & -6.594760 & -2.986225 & $\mathrm{I}(0)$ \\
\hline PO4 & $-3.990872^{*}$ & -2.981038 & -8.731717 & -2.986225 & $\mathrm{I}(0)$ \\
\hline
\end{tabular}




\section{International Journal of Engineering Applied Sciences and Technology, 2021 \\ Vol. 6, Issue 2, ISSN No. 2455-2143, Pages 17-25 \\ Published Online June 2021 in IJEAST (http://www.ijeast.com)}

\begin{tabular}{|l|l|l|l|l|l|}
\hline SO4 & $-5.972007^{*}$ & -2.981038 & -11.07675 & -2.986225 & $\mathrm{I}(0)$ \\
\hline $\mathrm{ZN}$ & $-4.623217^{*}$ & -2.981038 & -7.662919 & -2.986225 & $\mathrm{I}(0)$ \\
\hline
\end{tabular}

Source: Researcher's compilation from E-views 10 Regression output.

The Asterisks $(*)$ is used to indicate stationarity at the $5 \%$ level of significance.

From the stationarity test Table 4, only contamination distance (CONTD) achieved stationarity at first difference while; CA, CL, FE, MG, NA, NO3, PB, PO4, SO4 and ZN were stationary at level. Owing to the mixed order of stationarity, the Auto-regressive Distributed Lag (ARDL) model of estimation was adopted for further analysis.

Table 6. Correlation of Parameters

\begin{tabular}{|c|c|c|c|c|c|c|c|c|c|c|c|}
\hline & CONTD & $\mathrm{CA}$ & $\mathrm{CL}$ & $\mathrm{FE}$ & MG & NA01 & NO3 & PB & PO4 & $\mathrm{SO} 4$ & $\mathrm{ZN}$ \\
\hline CONTD & 1 & 0.0335 & 0.2952 & 0.088 & 0.3052 & -0.326 & -0.1088 & -0.2891 & -0.1183 & -0.1312 & 0.1241 \\
\hline$\overline{C A}$ & 0.0335 & 1 & 0.0532 & 0.3373 & -0.0866 & 0.1746 & $\begin{array}{l}-0.0473 \\
\end{array}$ & 0.2663 & 0.4018 & 0.0923 & -0.2455 \\
\hline $\mathrm{CL}$ & 0.2952 & 0.0532 & 1 & 0.2729 & 0.3549 & -0.5619 & 0.1993 & -0.1298 & -0.4438 & -0.366 & 0.1693 \\
\hline $\mathrm{FE}$ & 0.088 & 0.3373 & 0.2729 & 1 & 0.2244 & -0.1598 & 0.0547 & -0.1057 & 0.0183 & -0.1919 & -0.2533 \\
\hline$M G$ & 0.3052 & -0.0866 & 0.3549 & 0.2244 & 1 & -0.438 & 0.1739 & -0.1379 & -0.3196 & -0.1271 & 0.3227 \\
\hline NA01 & -0.326 & 0.17466 & -0.5619 & -0.1598 & -0.438 & 1 & -0.3254 & \begin{tabular}{|c|}
-0.1037 \\
\end{tabular} & 0.3722 & 0.3539 & -0.2851 \\
\hline $\mathrm{NO3}$ & -0.1088 & -0.0473 & 0.1993 & 0.0547 & 0.1739 & -0.3254 & 1 & -0.0631 & -0.2629 & -0.0266 & 0.3216 \\
\hline $\mathrm{PB}$ & -0.2891 & -0.2663 & -0.1298 & -0.1057 & -0.1379 & -0.1037 & -0.0631 & 1 & -0.0994 & 0.0176 & -0.1393 \\
\hline $\mathrm{PO} 4$ & -0.1183 & 0.4018 & -0.4438 & 0.0183 & -0.3196 & 0.3722 & -0.2629 & -0.0994 & 1 & -0.0764 & 0.0508 \\
\hline $\mathrm{SO} 4$ & -0.1312 & 0.0923 & -0.366 & -0.1919 & -0.1271 & 0.3539 & -0.0266 & 0.0176 & -0.0764 & 1 & -0.0471 \\
\hline $\mathrm{ZN}$ & 0.1241 & -0.2455 & 0.1693 & -0.2533 & 0.3227 & -0.2851 & 0.3216 & -0.1393 & 0.0508 & -0.0471 & 1 \\
\hline
\end{tabular}

From the Table 6, it can be seen that CA, CL, FE, MG and $\mathrm{ZN}$ have positive, but weak correlation with contamination distance as shown by their respective coefficients of $0.0335,0.2952,0.0880,0.3052$ and 0.1241 .

On the other hand, NA01, NO3, PB, PO4 and SO4 have negative, but weak correlation with contamination distance as shown by their respective coefficients of- $0.3260,-0.1088,-0.2891,-0.1183$ and -0.1312 .

Table 7 Parameters Output Estimation

Dependent Variable: (CONTD)

METHOD: ARDL

Dependent lags: 1 (Fixed)

Dynamic regressors (1 lag, fixed):

CA CL MG FE NA01 NO3 PB PO4 SO4 ZN

Fixed regressors: $\mathrm{C}$

\begin{tabular}{lllll}
\hline \hline Variable & Coefficient & Std. Error & t-Statistic & Prob.* \\
\hline \hline CONTD(-1) & 0.704701 & 0.234379 & 3.006677 & 0.0397 \\
CA & 0.147777 & 0.127021 & 1.163412 & 0.3093 \\
CA(-1) & 0.120883 & 0.143702 & 0.841208 & 0.4476 \\
CL & -1.194751 & 0.547932 & -2.180472 & 0.0947 \\
CL(-1) & -0.098453 & 0.415641 & -0.236870 & 0.8244 \\
MG & -1.619665 & 1.572963 & -1.029690 & 0.3613 \\
MG(-1) & -0.220989 & 1.231126 & -0.179502 & 0.8663 \\
FE & -13.31849 & 7.615823 & -1.748792 & 0.1552 \\
FE(-1) & 13.93407 & 9.300646 & 1.498183 & 0.2084 \\
NA01 & -0.185105 & 1.387117 & -0.133445 & 0.9003 \\
NA01(-1) & -3.971675 & 1.730264 & -2.295416 & 0.0834 \\
NO3 & 0.160229 & 0.606051 & 0.264383 & 0.8045 \\
NO3(-1) & -1.406493 & 0.707507 & -1.987957 & 0.1177 \\
PB & -292.6341 & 96.25526 & -3.040188 & 0.0384 \\
PB(-1) & -246.3712 & 130.1107 & -1.893550 & 0.1312
\end{tabular}




\section{International Journal of Engineering Applied Sciences and Technology, 2021 \\ Vol. 6, Issue 2, ISSN No. 2455-2143, Pages 17-25 \\ Published Online June 2021 in IJEAST (http://www.ijeast.com)}

\begin{tabular}{lllll} 
PO4 & -1.466277 & 1.331024 & -1.101616 & 0.3325 \\
PO4(-1) & -3.645375 & 1.699118 & -2.145451 & 0.0985 \\
SO4 & 0.277098 & 0.362318 & 0.764792 & 0.4870 \\
SO4(-1) & -0.170232 & 0.372139 & -0.457443 & 0.6711 \\
ZN & -6.788680 & 5.656026 & -1.200256 & 0.2963 \\
ZN(-1) & -0.906122 & 4.022297 & -0.225275 & 0.8328 \\
C & 211.4209 & 63.42219 & 3.333547 & 0.0290 \\
\hline \hline R-squared & 0.940456 & Mean dependent var & 83.84615 \\
Adjusted R-squared & 0.627850 & S.D. dependent var & 16.79212 \\
S.E. of regression & 10.24388 & Akaike info criterion & 7.311744 \\
Sum squared resid & 419.7484 & Schwarz criterion & 8.376287 \\
Log likelihood & -73.05267 & Hannan-Quinn criter. & 7.618294 \\
F-statistic & 3.008440 & Durbin-Watson stat & 2.822187 \\
Prob(F-statistic) & 0.146970 & & \\
\hline \hline
\end{tabular}

*Note: p-values and any subsequent tests do not account for model selection.

\section{$\underline{\text { Table 8. Cointegration Test }}$}

\begin{tabular}{lllll} 
F-Bounds Test & Null & \multicolumn{3}{c}{ Null Hypothesis: No levels relationship } \\
\hline \hline Test Statistic & Value & Signif. & $\mathrm{I}(0)$ & $\mathrm{I}(1)$ \\
\hline \hline F-statistic & 2.11 & $10 \%$ & 1.76 & 2.77 \\
K & 10 & $5 \%$ & 1.98 & 3.04 \\
& & $2.5 \%$ & 2.18 & 3.28 \\
& & $1 \%$ & 2.41 & 3.61 \\
\hline \hline
\end{tabular}

From the Table 8 above, it can be seen that there is no long run relationship in the model, but rather a short run phenomenon since the F-statistic value of 2.11 is greater than the $I(0)$ value, but less than the $I(1)$ value; hence the absence of long run relationship in the model as revealed by the Bounds test.

Table 9: Statistical derivation of contamination distance using SPSS and E-View.

Dependent Variable: (CONTD)

Selected Model: ARDL(1, 1, 1, 1, 1, 1, 1, 1, 1, 1, 1)

Case 2: Restricted Constant and No Trend

Date: 08/12/20 Time: 15:27

Sample: 127

Included observations: 26

ECM Regression

Case 2: Restricted Constant and No Trend

\begin{tabular}{lllll}
\hline \hline Variable & Coefficient & Std. Error & t-Statistic & Prob. \\
\hline \hline D(CA) & 0.147 & 0.031 & 4.642 & 0.0097 \\
$D(C L)$ & -1.194 & 0.154 & -7.72 & 0.0015 \\
D(FE) & -13.318 & 2.065 & -6.44 & 0.0030 \\
D(MG) & -1.619 & 0.431 & -3.75 & 0.0199
\end{tabular}




\section{International Journal of Engineering Applied Sciences and Technology, 2021 \\ Vol. 6, Issue 2, ISSN No. 2455-2143, Pages 17-25 \\ Published Online June 2021 in IJEAST (http://www.ijeast.com)}

\begin{tabular}{lllll} 
D(NA01) & -0.185 & 0.476 & -0.38 & 0.7177 \\
D(NO3) & 0.160 & 0.173 & 0.921 & 0.4089 \\
D(PB) & -292.63 & 41.06 & -7.12 & 0.0020 \\
D(PO4) & -1.466 & 0.417 & -3.50 & 0.0247 \\
D(SO4) & 0.277 & 0.113 & 2.440 & 0.0712 \\
D(ZN) & -6.788 & 1.220 & -5.56 & 0.0051 \\
CointEq(-1)* & -0.295 & 0.030 & -9.75 & 0.0006 \\
\hline \hline R-squared & 0.928 & Mean dependent var & 0.076 \\
Adjusted R-squared & 0.881 & S.D. dependent var & 15.34 \\
S.E. of regression & 5.289 & Akaike info criterion & 6.465 \\
Sum squared resid & 419.7 & Schwarz criterion & 6.997 \\
Log likelihood & -73.0 & Hannan-Quinn criter. & 6.618 \\
Durbin-Watson stat & 2.822 & \multicolumn{3}{c}{} \\
\hline \hline
\end{tabular}

* p-value incompatible with t-Bounds distribution.

The prediction model for calculating effect of distance from contaminants is obtained as follows:

$$
\begin{aligned}
D(C O N T D)= & 0.147 D(C A)-0.194 D(C L)-13.318 D(F E)-1.619 D(M G)+0.160 D(N O 3)-292.63 D(P B) \\
& -1.466 D(P O 4)+0.277 D(S O 4)-6.788 D(Z N) \\
& -0.295 \text { CointEq(-1)** } *^{*}
\end{aligned}
$$

Substituting the parameters into equation 1, we obtain the value for the minimum allowable distance for locating a borehole from a septic tank as shown below.

Table 10 below shows the values of distance when these chemical parameters were substituted in the predicting model (equation 1). It can be seen that the lowest value is 9.214 at Isiala Mbano while the highest is 26.19 at Okigwe LGA. The overall mean or average of these distances shows that $15.81 \mathrm{~m}$ is the minimum value for location of septic tank near boreholes in Okigwe zone.

Table 10: Computation of Contamination Distance for Okigwe zone

\begin{tabular}{|l|lll|l|l|l|l|l|l|l|l|l|}
\hline LGA & $\mathrm{Ca}$ & $\mathrm{Zn}$ & $\mathrm{Pb}$ & $\mathrm{Fe}$ & $\mathrm{Mg}$ & $\mathrm{Na}$ & $\mathrm{SO} 4$ & $\mathrm{PO} 4$ & $\mathrm{CL}$ & $\mathrm{NO} 3$ & Distance & ABS(D) \\
\hline Ehime & 41.2 & 0.8 & 0 & 0.43 & 3.43 & 2.48 & 3.5 & 5 & 12.4 & 7 & -18.6 & 18.6 \\
\hline & 57.8 & 0.7 & 0 & 0.29 & 5.04 & 0 & 5.1 & 2.8 & 11.7 & 3.5 & -12.97 & 12.97 \\
\hline & 53.4 & 0.6 & 0 & 0.8 & 0.04 & 1.55 & 2.6 & 2.3 & 9.3 & 4.6 & -10.96 & 10.96 \\
\hline & 39.6 & 1.1 & 0.02 & 0.22 & 2.02 & 1.59 & 0 & 3.4 & 7.6 & 5.9 & -19.51 & 19.51 \\
\hline Isiala & 33 & 1.2 & 0 & 0.16 & 3.05 & 2.02 & 6.3 & 0 & 4.6 & 3.7 & -9.214 & 9.214 \\
\hline & 35.2 & 1.1 & 0 & 0.38 & 2.9 & 2.32 & 11.9 & 5.1 & 5.4 & 3.8 & -16.96 & 16.96 \\
\hline & 37.8 & 0.8 & 0.15 & 0.2 & 1.28 & 0 & 12.7 & 4.8 & 6.3 & 2.7 & -28.11 & 28.11 \\
\hline & 40.8 & 0.7 & 0 & 0.4 & 1.39 & 2.04 & 2.1 & 1.7 & 6.8 & 4 & -9.216 & 9.216 \\
\hline Onuim & 40.2 & 0.7 & 0 & 0.4 & 1.85 & 3.23 & 2.9 & 0 & 6.4 & 5.2 & -7.066 & 7.066 \\
\hline & 42.4 & 0.5 & 0 & 0.32 & 1.04 & 4.01 & 11.8 & 2.6 & 5.6 & 3.2 & -4.519 & 14.519 \\
\hline & 32.2 & 0.6 & 0 & 0.3 & 2.05 & 2.01 & 2.7 & 3.8 & 6.2 & 7.2 & -11.82 & 11.82 \\
\hline & 34.6 & 1 & 0 & 0.29 & 1.06 & 0 & 3.6 & 4.7 & 4.8 & 6.3 & -13.39 & 13.39 \\
\hline Obow & 37.2 & 0.7 & 0 & 0.77 & 4.09 & 0 & 3.6 & 4.4 & 7.2 & 4.8 & -22.54 & 22.54 \\
\hline & 38.4 & 1.1 & 0 & 0.42 & 3.2 & 2.34 & 0 & 3.6 & 12.5 & 3.7 & -20 & 20 \\
\hline & 42.6 & 1 & 0.02 & 0.36 & 1.84 & 3.12 & 2.4 & 3.2 & 17.4 & 11 & -20.09 & 20.09 \\
\hline & 44.8 & 0.7 & 0 & 0.38 & 3.24 & 3.41 & 13.2 & 3.4 & 8.2 & 7.2 & -10.53 & 10.53 \\
\hline Ihitte & 41.8 & 0.8 & 0 & 0.9 & 2.44 & 4.05 & 0 & 3.3 & 6.7 & 4.8 & -20.89 & 20.89 \\
\hline & 53.4 & 1.2 & 0.01 & 0.8 & 1.82 & 2.84 & 12.6 & 0 & 8.2 & 3.7 & -16.09 & 16.09 \\
\hline & 37 & 1.4 & 0 & 0.45 & 3.04 & 0 & 13.2 & 4.4 & 7.9 & 4.4 & -18.9 & 18.9 \\
\hline & 38.6 & 1 & 0 & 0.32 & 4.31 & 1.96 & 14.1 & 3.4 & 8.8 & 5.7 & -14.52 & 14.52 \\
\hline Okigw & 29.2 & 0.8 & 0 & 0.4 & 2.43 & 1.34 & 6.7 & 0 & 10.3 & 6.8 & -9.749 & 9.749 \\
\hline
\end{tabular}


International Journal of Engineering Applied Sciences and Technology, 2021

Vol. 6, Issue 2, ISSN No. 2455-2143, Pages 17-25

Published Online June 2021 in IJEAST (http://www.ijeast.com)

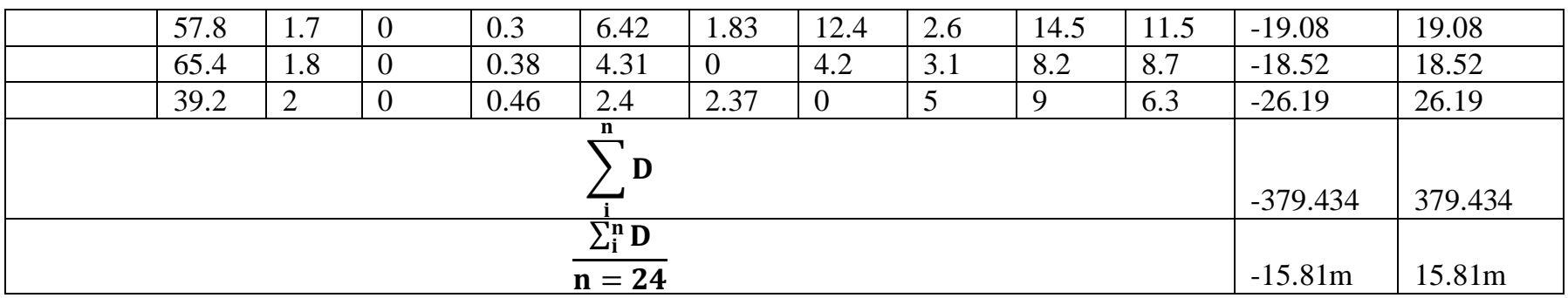

Base on the lowest value and highest value and their results, it can be seen that the results have significant difference as shown below for chemical parameters. The values for $\mathrm{Pb}$ and nitrate for the two samples before treatment were $0.02,11.8$, and Nil, 3.7 respectively. This shows that the nearer the borehole is to the septic tank the chances of contamination is higher and vice versa.

The chemical parameters values for calcium, magnesium, sodium and zinc were within permissible limits. Lead $(\mathrm{Pb})$ contaminations with values ranging between 0.015 to 0.04 were observed which were above $0.01 \mathrm{mg} / \mathrm{l}$ standard permitted by NSDQW and WHO for portable drinking water. Lead contamination has been reported to have adverse health implication which includes cancer, vitamin D metabolism interference, impairment of proper infant mental development, toxicity to the central and peripheral nervous systems (NSDQW, 2007). Foster et al., (2002) reported associated lead contamination source as also possible from septic tanks and pit latrine. Iron contamination with values higher than $0.3 \mathrm{mg} / \mathrm{l}$ were recorded which could be as a result of steel pipes. The possible health effects are high concentration of iron stored in the pancreas, liver, spleen (Oteze, 1991). High concentration of iron in the body can cause liver and lung problems (Offodile, 1987). Values of sulphate below the WHO and NSDWQ stipulated levels were observed in all the samples. High levels of sulphate in drinking water can lead to dehydration and diarrhea especially in children (NSDWQ, 2007). It can also cause noticeable taste and very high levels might cause laxative effect in unaccustomed consumers. Phosphate are not toxic to people or animals unless when they are present in very high levels.

Longe and Balogun (2010) associated high phosphate levels in ground water as due to landfill operations and fertilizer application on farm lands. Excess chloride can be dangerous to the health of both humans and farm animals. Some samples show nitrate values that exceed the recommended value of $10 \mathrm{mg} / \mathrm{l}$ by WHO. Nitrate could come from municipal and industrial waste water including leach from waste disposal system (Foster et al., 2002). High nitrate concentrations have detrimental effects on infants less than 3.6 months of age and can also lead to blue baby disease or syndrome which threatens the oxygen carrying capacity of the blood around the body (Chapman, 1996).

Tables 3 to 5 show increase of some chemical parameters' like calcium, iron, magnesium, sodium, phosphate etc after treatment. On the contrary, values of zinc, sulphate, nitrate and lead were reduced significantly from their original values after treatment. These observations correspond to the views of Luca (2007).

The minimum allowable distance between septic tank and borehole for contaminants was calculated to be 15.81metres. This means that in Imo state, it is safe to site borehole at a distance from $15 \mathrm{~m}$ and above from septic tanks. This conforms to standard engineering practice.

\section{CONCLUSION}

The overall importance of this study is that it is no longer speculation that Imo state groundwater needs some levels of treatment before use /consumption. The level of treatment has passed use of net and alum. The three treatment methods were able to treat the samples with different degrees of efficiency. Some samples reduced in concentration while some increased in concentration. There are heavy consequences of ignoring the health implication of Imolites by paying less attention to regulation of drinking water. The minimum distance for location of borehole from septic tank is $15.81 \mathrm{~m}$ from the prediction model.

\section{REFERENCES}

1 Chapman, D. (1996) Water Quality Assessment- A guide to use of Biota, Sediments and water in Environmental Monitoring $2^{\text {nd }}$ Ed.UNESCO/WHO/UNEP,Chapman and Hall,London Pp651. 


\section{International Journal of Engineering Applied Sciences and Technology, 2021 Vol. 6, Issue 2, ISSN No. 2455-2143, Pages 17-25 \\ Published Online June 2021 in IJEAST (http://www.ijeast.com)}

2 Chessbrough, M. (2014): District Laboratory Practice inTropical Countries, part 2. Cambriedge Universersity Press, cambrdge 140.

3 Feachem, R., Chem, M.,and Duncen,M.(1998). Wastewater and Health in hot climates. John Whiley and sons chichester, $4^{\text {th }}$ Ed.Pp164

4 Ijeh, B.I and Onu, N.N (2013): Assessment of Pollution Levels of Groundwater in Parts of Imo River Basin. South - Eastern, Nigeria. International Jour. Of Water Resources and Environmental Engineering 5(4): 194-202.

5 Longe, E.O. and Balogun,M.R.(2010). Groundwater Quality Assessment near a municipal landfill, Lagos, Nigeria. Research Journal of Applied Sciences, Engineering and Technology2(1)39-44.

6 Luca, G. (2007): Review of Zero Valent Iron and Apitite as Reactive Materials for Permeable Reactive Barrier. Dept. of Environmental Sciences Zurich

7 Nickson,R.T. McArthur, J.M., Shrestha B, Kyaw-Myint, T.O and Lowry, D.(2005): Arsenic and other drinking water quality issues. Muzaffargarh District. Pakistan . Appl.Geochem.20(1):55

8 NSDWQ (2007): Nigerian Standard for Drinking Water Quality, Nigeria Industrial Standard(NIS) 554. Standard Organization of Nigeria.

9 Nwachukwu M.A., (2014): Prospective Techniques for in-situ Treatment and protection of Aquifers: A sustainable Hydrological Review. Dept of Environmental Technology FUTO. Inter. Jour. Of Water Resources and Environ. Engineering.

10 Offodile,M.E.(1987) Water resources management and the Nigerian groundwater system. Proceedings of delegate conference of the Nigeria Academy of science,Pp 2335.

11 Oteze, G.E.(1991). Potability of Groundwater from Rima Group Aquifers in the Sokoto Basin. J.Min. Geol.27(1):17-34

12 Punmia,B.C. and Jain,A.K.(1998). Wastewater Engineering Laxim publication(p) ltd,New DelhiPp155.

13 Rajasulocham,P and V. Preethy(2016): Comparison on Efficiency of various Techniques in treatment of waste and sewage water- A comprehensive Review. Crossmark, Chennai India.
14 Ramakrishnaiah CR, Sadashivaiah C, Ranganna G (2009). Assessment of Water Quality Index for the Groundwater in Tumkur Taluk, Karnataka State, India. E-J. Chem. 6(2):523-530. 